\title{
11 RECONSTRUÇÃO DA IDENTIDADE PESSOAL NA DOENÇA CRÓNICA: UMA REVISÃO INTEGRATIVA
}

\author{
| Helga Rafael ${ }^{1} \mid$
}

\section{RESUMO}

Viver com doença crónica afeta a identidade pessoal. O objetivo deste artigo é rever os estudos empíricos e teóricos sobre como a experiência de conviver com doença crónica afeta a identidade pessoal. Não se conhece nenhuma revisão da literatura sobre este assunto. Realizou-se uma revisão integrativa da literatura, tendo-se procedido à análise de 12 estudos. Os resultados mostram que as pessoas procuram naturalmente definir e expressar a sua identidade pessoal. A análise da amostra bibliográfica permitiu identificar três categorias: Elementos identitários postos em causa na doença crónica; Sentimento de si na doença crónica; e Fases da (Re)construção da Identidade Pessoal. Vários elementos identitários, como o grau de atividade, as capacidades físicas ou o grau de dependência, ficam perturbados, podendo conduzir a sentimentos de perda, estranheza, insegurança ou isolamento. A pessoa percorre diferentes fases até à reconstrução da sua identidade. É necessária mais investigação para clarificar este processo.

PALAVRAS-CHAVE: Saúde mental; Doença crónica; Enfermagem

\section{RESUMEN}

"Reconstrucción de la identidad personal en la enfermedad crónica: Una revisión integradora"

Vivir con la enfermedad crónica afecta a la identidad personal. El propósito de este artículo es revisar los estudios empíricos y teóricos sobre como la experiencia de vivir con una enfermedad crónica afecta a la identidad personal. No se conoce ninguna revisión de la literatura sobre este tema. Hemos llevado a cabo una revisión integradora de la literatura y se procedió al análisis de 12 estudios. Los resultados muestran que las personas buscan naturalmente, definir y expresar su identidad personal. El análisis de la muestra de la literatura identificó tres categorías: Elementos de identidad cuestionados en la enfermedad crónica; Sintiéndose en la enfermedad crónica; Etapas de (re)construcción de la identidad personal. Varios elementos de identidad, tales como el grado de actividad, la capacidad física o el grado de dependencia, son perturbados y pueden conducir a sentimientos de pérdida, la torpeza, la inseguridad o el aislamiento. La persona pasa por diferentes fases de la reconstrucción de sua identidad. Se necesita más investigación para aclarar este proceso.

DESCRIPTORES: Salud mental; Enfermedad crónica; Enfermería

\begin{abstract}
"Personal identity rebuilding in chronic illness: An Integrative Review”

Living with chronic illness affects one's personal identity. The purpose of this article is to review empirical and theoretical studies about how the experience of living with chronic illness affects personal identity. There is no known literature review on this subject. An integrative review of the literature was carried out and 12 studies were analysed. The results show that people naturally seek to define and express their personal identity. The analysis of the bibliographic sample allowed to identify three categories: Identity elements questioned chronic disease; Sense of self in chronic illness; And Phases of (re)construction of personal identity. Identity elements, such as degree of activity, physical capacities or degree of dependence, are disturbed and can lead to feelings of loss, strangeness, insecurity or isolation. The person goes through different phases until the reconstruction of a new identity. More research is needed to clarify this process.
\end{abstract}

\section{KEYWORDS: Identity; Chronic disease; Nursing}

Submetido em 06-01-2017 Aceite em 29-10-2017

\footnotetext{
$1 \mathrm{PhD}$ in Nursing; MSc in Nursing; Postgraduate Diploma in Medical-Surgical Nursing; Nursing Diploma; Researcher at Escola Superior de Enfermagem de Lisboa, Unit of Research \& Development in Nursing; Assistant Professor at Escola Superior de Enfermagem de Lisboa, Av. Prof. Egas Moniz, 1600-190 Lisboa, Portugal, hrafael@esel.pt 


\section{INTRODUÇÃO}

As pessoas com doença crónica - utilizadores frequentes dos serviços de saúde - têm muitas vezes associados problemas de saúde mental (EUROPEAN UNION [EU], 2016). As mais recentes orientações recomendam um reconhecimento precoce das desordens mentais destas pessoas a fim de desenvolver ações que possam contribuir para melhorar a saúde, a qualidade de vida e resiliência, reduzindo a adesão a comportamentos aditivos, à inatividade física e à má alimentação (EU, 2016). Para este reconhecimento é imperativo um acesso à perceção que cada pessoa tem de si.

Identidade pessoal é a compreensão que cada um faz de si, incluindo as suas habilidades, valores, posição social, sentido de vida e impacto nos outros. A identidade pessoal deve ser entendida como algo inacabado, que se vai construindo ao longo da vida e na interação com o outro. Representa aquilo que cada um atribuí para si mesmo como sendo aceitável e congruente com o seu passado e com o que espera ser o seu futuro (Nordenfelt, 2009). É através da identidade que a pessoa se define e se distingue dos outros, o que se reflete nas preferências, desejos, esperança e planos para o futuro. Representa, por isso, a fronteira entre o indivíduo e a sociedade (Karnilowicz, 2011). Trata-se, portanto, de um conceito ligado à autoperceção de integralidade que, quando perturbada, compromete a saúde (Herdman \& Kamitsuru, 2014), transformando-se em doença mental (Mathews, 2013).

O sentimento da identidade pessoal está fortemente dependente de sentimentos de autoconfiança, autorrespeito, autoestima e autoimagem (Nordenfelt, 2009). Está ligado a conceitos como autonomia (Dale, Soderhamn, \& Soderhamn, 2012), dignidade e respeito (Dale et al., 2012).

Identidade pessoal é um conceito que tem merecido uma atenção crescente na área da saúde nos últimos tempos. A revisão da produção científica no âmbito dos cuidados de enfermagem mostra que o conceito identidade pessoal nem sempre é clarificado, surgindo muitas vezes apenas nomeado com sentidos equivalentes ou próximos do conceito de pessoa (Rafael, 2012). A identidade pessoal é um conceito multidimensional que, tal como o conceito de pessoa, integra as dimensões individual, profissional, familiar, social e de lazer.

Para Meleis, Sawyer, Im, Messias, \& Schumacher (2000) a identidade pessoal sofre mudanças importantes nos momentos de transição, tornando-se integra e fluida quando ocorre uma transição saudável.
No entanto, pouco se conhece acerca do processo de redefinição de uma identidade congruente com uma transição saudável.

O objetivo deste artigo é, deste modo, rever os estudos empíricos e teóricos sobre como a experiência de conviver com doença crónica afeta a identidade pessoal. Embora alguns investigadores apontem para a necessidade de investigar a identidade pessoal na doença crónica (Henriques, 2015; Luyckx, et al., 2008), não é conhecida nenhuma revisão sistemática ou integrativa sobre este assunto. Este fenómeno ganha um maior interesse na disciplina de enfermagem quando percebemos que vários referenciais teóricos salientem a necessidade de se reconhecer a experiência de saúde subjetiva e singular de cada pessoa para desenvolver cuidados de enfermagem holísticos (Meleis et al., 2000) e, assim, respeitando a sua identidade pessoal.

\section{MÉTODOS}

A revisão integrativa da literatura é considerada a mais ampla abordagem metodológica referente às revisões da literatura. Permite, por isso, uma compreensão abrangente do fenómeno em estudo, recorrendo a estudos experimentais e não experimentais e a dados da literatura teórica e empírica (Souza, Silva, \& Carvalho, 2010). Com o propósito de identificar artigos empíricos e teóricos publicados sobre a identidade pessoal em relação com a experiência de convivência com a doença crónica na pessoa adulta ou idosa, realizou-se uma pesquisa online nas bases de dados Cumulative Index to Nursing and Allied Health Literature (CINAHL) e Medical Literature Analysis and Retrieval System Online (MEDLINE).

Tal como Souza et al. (2010) defendem recorreu-se também à utilização de material não-publicado para garantir uma maior representatividade da amostra, através do acesso ao Repositório Científico de Acesso Aberto de Portugal (RCAAP).

As pesquisas foram realizadas com restrições de data nos últimos 10 anos (2006-2016), utilizando como palavras-chave identidade pessoal, personal identity, self-identity, identity, doença crónica, chronic illness, chronic disease, Chronically Ill, long-term disease.

Nas bases de dados CHINAL e MEDLINE pesquisouse individualmente em cada base de dados, utilizando a seguinte estratégia: [TI(Personal identity OR selfidentity OR identity)] AND [AB OR SU(chronic illness OR chronic disease OR Chronically Ill OR long-term disease)]. 
Obteve-se 70 artigos nas duas bases de dados, sendo que apenas 42 estavam disponíveis em texto completo. A análise foi canalizada apenas para os artigos referentes à pessoa adulta ou idosa e eliminaram-se 11 artigos. Dos 31 artigos restantes, foram eliminados os que estavam repetidos, tendo ficado 23 artigos. Eliminaram-se 5 artigos com data inferior a 2006. A análise do texto integral permitiu eliminar 7 artigos por não se enquadrarem no estudo do fenómeno em análise. Obteve-se 11 artigos. No RCAAP utilizámos uma estratégia semelhante: [Título (Identidade pessoal OR Personal identity OR self-identity OR identity)] AND [Descrição (doença crónica OR chronic illness OR chronic disease OR Chronically Ill OR long-term disease)]. Identificouse apenas um documento, que integrou a amostra final. Após a extração dos dados dos artigos selecionados, procedeu-se à análise categorial dos mesmos, ponderando o rigor de cada estudo.
Uma das categorias - fases de reconstrução da identidade pessoal - foi definida à priori e descrita com base no Modelo Transteórico de Mudança de Comportamento (Prochaska, Redding, \& Evers, 2008).

\section{RESULTADOS E DISCUSSÃO}

A amostra bibliográfica final foi constituída por 12 estudos empíricos, envolvendo 538 pessoas com doença crónica. As investigações foram conduzidas em países como os EUA $(n=5)$, o Reino Unido $(n=5)$, a Bélgica $(n=1)$ e Portugal $(n=1)$. Três estudos tinham uma abordagem quantitativa e os restantes 9 eram baseados numa abordagem qualitativa, com participantes com cancro coloretal, cancro da mama, cancro da próstata, artrite reumatoide, diabetes tipo 1, HIV-positivo, doença respiratória crónica, cancro do estômago e cancro do pulmão, entre outras. Todos os estudos têm como foco a identidade pessoal na experiência de conviver com uma doença crónica (Quadro 1).

Quadro 1 - Estudos que Integram a Revisão Integrativa da Literatura

\begin{tabular}{|c|c|c|c|c|c|}
\hline Estudo & País & Participantes & Objetivo & $\begin{array}{l}\text { Tipo de } \\
\text { estudo }\end{array}$ & Quadro concetual \\
\hline $\begin{array}{l}\text { Hubbard, G., Kidd, L., } \\
\text { \& Kearney, N. (2010). }\end{array}$ & $\begin{array}{l}\text { Reino } \\
\text { Unido }\end{array}$ & $\begin{array}{l}18 \text { pessoas com } \\
\text { cancro colo-retal }\end{array}$ & $\begin{array}{l}\text { Explorar em que medida as pessoas experienciam o } \\
\text { cancro como disrupção biográfica no primeiro ano } \\
\text { após o diagnóstico. }\end{array}$ & $\begin{array}{l}\text { Estudo } \\
\text { longitudinal } \\
\text { qualitativo }\end{array}$ & $\begin{array}{l}\text { Teoria da disrupção } \\
\text { biográfica }\end{array}$ \\
\hline Karnilowicz, W. (2011). & EUA & $\begin{array}{l}1 \text { pessoa com } \\
\text { cancro da próstata } \\
\text { e doença crônica. }\end{array}$ & $\begin{array}{l}\text { Explorar a propriedade psicológica e identidade rela- } \\
\text { cionada com cancro da próstata e doença crônica. }\end{array}$ & $\begin{array}{l}\text { Auto-etno- } \\
\text { grafia }\end{array}$ & $\begin{array}{l}\text { Construtivismo } \\
\text { social. }\end{array}$ \\
\hline Luyckx, et al.. (2008). & Bélgica & $\begin{array}{l}194 \text { Jovens-adultos } \\
\text { com diabetes tipo } \\
1 \text { e } 344 \text { não diabé- } \\
\text { ticos. }\end{array}$ & $\begin{array}{l}\text { Examinar em que medida o desenvolvimento da iden- } \\
\text { tidade foi afetado por ter diabetes, comparando com o } \\
\text { desenvolvimento numa amostra de não-diabéticos; } \\
\text { Examinar como a identidade se relaciona com sintomas } \\
\text { depressivos, coping com a diabetes, e problemas rela- } \\
\text { cionados com a diabetes na amostra de diabéticos; } \\
\text { Examinar em que medida os percursos do desenvolvi- } \\
\text { mento da identidade para problemas com diabetes e } \\
\text { sintomas depressivos são mediados por estratégias de } \\
\text { coping na amostra de diabéticos. }\end{array}$ & \begin{tabular}{|l} 
Estudo \\
quantitativo \\
Corte trans- \\
versal
\end{tabular} & $\begin{array}{l}\text { Teoria do desen- } \\
\text { volvimento psicos- } \\
\text { social }\end{array}$ \\
\hline $\begin{array}{l}\text { Golub, S. A., Rendina, } \\
\text { H. J., \& Gamarel, K. E. } \\
\text { (2013). }\end{array}$ & EUA & $\begin{array}{l}129 \text { homens HIV- } \\
\text { positivo }\end{array}$ & $\begin{array}{l}\text { Descrever o desenvolvimento e a pilotagem de um } \\
\text { novo instrument, designado Impact on Self Concept } \\
\text { Scale (ISCS). }\end{array}$ & $\begin{array}{l}\text { Estudo } \\
\text { quantitativo } \\
\text { Corte trans- } \\
\text { versal }\end{array}$ & $\begin{array}{l}\text { Evidência sobre } \\
\text { crescimento pós- } \\
\text { traumático }\end{array}$ \\
\hline $\begin{array}{l}\text { Golub, S. A., Gamarel, } \\
\text { K. E., \& Rendina, H. J. } \\
\text { (2014). }\end{array}$ & EUA & $\begin{array}{l}60 \text { pessoas HIV- } \\
\text { positivo }\end{array}$ & $\begin{array}{l}\text { Examinar a influência do autocrescimento na perceção } \\
\text { de perda de saúde física e mental entre as pessoas que } \\
\text { vivem com HIV. }\end{array}$ & $\begin{array}{l}\text { Estudo } \\
\text { quantitativo }\end{array}$ & $\begin{array}{l}\text { Evidência sobre } \\
\text { crescimento pós- } \\
\text { traumático }\end{array}$ \\
\hline Shepherd, M. (2010). & $\begin{array}{l}\text { Reino } \\
\text { Unido }\end{array}$ & $\begin{array}{l}31 \text { pessoas com } \\
\text { diabetes monogé- } \\
\text { nica. }\end{array}$ & $\begin{array}{l}\text { Explorar as experiências de doentes identificados com } \\
\text { diabetes monogénica e o impacto da transferência de } \\
\text { insulina para sulfoniluréias }\end{array}$ & $\begin{array}{l}\text { Estudo } \\
\text { qualitativo } \\
\text { longitudinal }\end{array}$ & $\begin{array}{l}\text { Teoria da disrupção } \\
\text { biográfica }\end{array}$ \\
\hline $\begin{array}{l}\text { Guise, J., McKinlay, } \\
\text { A., \& Widdicombe, S. } \\
(2010) \text {. }\end{array}$ & $\begin{array}{l}\text { Reino } \\
\text { Unido }\end{array}$ & $\begin{array}{l}12 \text { pessoas com } \\
\text { doença cónica } \\
5 \text { cuidadores } \\
\end{array}$ & $\begin{array}{l}\text { Examinar o modo como os doentes falam sobre o AVC } \\
\text { precoce e sobre os efeitos que esta doença crónica teve } \\
\text { na identidade. }\end{array}$ & Focus grupo & $\begin{array}{l}\text { Construtivismo } \\
\text { social }\end{array}$ \\
\hline $\begin{array}{l}\text { Baumgartner, L. M. } \\
\text { (2007). }\end{array}$ & EUA & $\begin{array}{l}18 \text { pessoas HIV- } \\
\text { positivo ( } 10 \text { ho- } \\
\text { mens e } 8 \text { mulheres) }\end{array}$ & $\begin{array}{l}\text { Examinar como as pessoas incorporam a identidade } \\
\text { HIV nos seus selves em três momentos. }\end{array}$ & $\begin{array}{l}\text { Estudo } \\
\text { qualitativo }\end{array}$ & $\begin{array}{l}\text { Evidência sobre o } \\
\text { efeito da doença } \\
\text { crónica na identi- } \\
\text { dade }\end{array}$ \\
\hline
\end{tabular}




\begin{tabular}{|c|c|c|c|c|c|}
\hline $\begin{array}{l}\text { Lempp, H., Scott, D., \& } \\
\text { Kingsley, G. (2006). }\end{array}$ & $\begin{array}{l}\text { Reino } \\
\text { Unido }\end{array}$ & $\begin{array}{l}26 \text { pessoas com } \\
\text { artrite reumatoide. }\end{array}$ & $\begin{array}{l}\text { Compreender a experiência pessoal de viver com } \\
\text { artrite reumatoide e o impacto da doença na vida dos } \\
\text { doentes. }\end{array}$ & $\begin{array}{l}\text { Estudo } \\
\text { qualitativo }\end{array}$ & $\begin{array}{l}\text { Construtivismo } \\
\text { social }\end{array}$ \\
\hline $\begin{array}{l}\text { Adams, W. E., Todor- } \\
\text { ova, I. G., Guzzardo, } \\
\text { M. T., \& Falcón, L. M. } \\
(2015) \text {. }\end{array}$ & EUA & $\begin{array}{l}30 \text { pessoas com } \\
\text { doença crónica. }\end{array}$ & $\begin{array}{l}\text { Compreender a forma como significados pessoais e } \\
\text { multiculturais sobre medicação estão relacionados e } \\
\text { integrados na identidade. }\end{array}$ & $\begin{array}{l}\text { Estudo } \\
\text { longitudinal } \\
\text { qualitativo }\end{array}$ & $\begin{array}{l}\text { Evidência sobre } \\
\text { adesão à medicação } \\
\text { e significados }\end{array}$ \\
\hline $\begin{array}{l}\text { Reynolds, F., \& Prior, S. } \\
\text { (2006). }\end{array}$ & $\begin{array}{l}\text { Reino } \\
\text { Unido }\end{array}$ & $\begin{array}{l}2 \text { mulheres com } \\
\text { cancro da mama e } \\
1 \text { homem com can- } \\
\text { cro no estomago e } \\
\text { no pulmão }\end{array}$ & $\begin{array}{l}\text { Compreender porquê que algumas pessoas com cancro } \\
\text { assumem a arte como atividade de lazer e como a arte } \\
\text { visual no dia-a-dia pode suportar a manutenção/recon- } \\
\text { strução da identidade. }\end{array}$ & $\begin{array}{l}\text { Estudo } \\
\text { fenom- } \\
\text { enológico }\end{array}$ & $\begin{array}{l}\text { Evidência sobre o } \\
\text { efeito da doença } \\
\text { crónica na identi- } \\
\text { dade e no self. }\end{array}$ \\
\hline Henriques, H. (2015). & $\begin{array}{l}\text { Portu- } \\
\text { gal }\end{array}$ & $\begin{array}{l}16 \text { pessoas com } \\
\text { DPOC }\end{array}$ & $\begin{array}{l}\text { Compreender de que modo a pessoa idosa que vive } \\
\text { com DPOC em sua casa é afetada pela experiência de } \\
\text { autocuidado na sua identidade pessoal }\end{array}$ & $\begin{array}{l}\text { Estudo } \\
\text { fenom- } \\
\text { enológico }\end{array}$ & Fenomenologia \\
\hline
\end{tabular}

Os participantes foram recrutados de vários locais, incluindo consultórios médicos, consultas hospitalares de especialidade, laboratórios, grupos de suporte, grupos de terapia ocupacional, registos nacionais de doentes, associações de doentes e comunidade.

A análise da amostra bibliográfica permitiu identificar um conjunto de três grandes categorias que integram o tema 'Identidade pessoal na doença crónica': Elementos identitários postos em causa na doença crónica; Sentimento de si na doença crónica; e Fases de (re)construção da identidade pessoal (Quadro 2).

Quadro 2 - Categorias e Subcategorias que Integram o Tema 'Identidade Pessoal na Doença Crónica'

\begin{tabular}{|l|l|l|l|}
\hline CATEGORIA & $\begin{array}{l}\text { Elementos identitários postos } \\
\text { em causa na doença crónica }\end{array}$ & Sentimento de si na doença crónica & $\begin{array}{l}\text { Fases de (re)construção da iden- } \\
\text { tidade pessoal }\end{array}$ \\
\hline SUB-CATEGORIA & $\begin{array}{l}\text { Grau de Atividade } \\
\text { Capacidades físicas } \\
\text { Grau de dependência } \\
\text { Disposição emocional } \\
\text { Autoestima } \\
\text { Autoimagem }\end{array}$ & $\begin{array}{l}\text { Sentimento de perda } \\
\text { Sentimento de estranheza: 'o outro eu' } \\
\text { Sentimento de insegurança } \\
\text { Sentimento de luta permanente pela } \\
\text { normalidade } \\
\text { Sentimento de isolamento }\end{array}$ & $\begin{array}{l}\text { Identidade antiga } \\
\text { Ameaça à identidade } \\
\text { Negociação identitária } \\
\text { (Des/re)construção da identidade } \\
\text { Identidade reconstruída }\end{array}$ \\
& & & \\
\end{tabular}

\section{Elementos Identitários Postos em Causa na Doença Crónica}

A pessoa com doença crónica está sujeita a uma experiência de mudança que coloca em causa diferentes dimensões da identidade pessoal. Existe a necessidade de reconfigurações nos elementos identitários grau de atividade, capacidades físicas, grau de dependência, disposição emocional, autoestima, autoimagem, autoconceito e papel social (Quadro 3).

Quadro 3 - CATEGORIA: Elementos Identitários Postos em Causa na Doença Crónica

\begin{tabular}{|l|l|}
\hline SUBCATEGORIA & \\
\hline Grau de Atividade & $\begin{array}{l}\text { Há uma transição forçada de uma vida ativa para uma vida passiva (Henriques, 2015; Lempp } \\
\text { et al., 2006); }\end{array}$ \\
\hline Capacidades físicas & $\begin{array}{l}\text { Verifica-se uma progressiva perda de capacidades físicas (Henriques, 2015; Hubbard et al., } \\
\text { 2010; Lempp et al., 2006); }\end{array}$ \\
\hline Grau de dependência & Há um aumento da dependência (Henriques, 2015; Hubbard et al., 2010; Lempp et al., 2006). \\
\hline Disposição emocional & Há alterações na disposição emocional (Hubbard et al., 2010; Lempp et al., 2006). \\
\hline Autoestima & Alterações na autoestima (Henriques, 2015). \\
\hline Autoimagem & Alterações na autoimagem (Henriques, 2015; Hubbard et al., 2010). \\
\hline Autoconceito & Alterações no autoconceito (Golub at al., 2014). \\
\hline Papel Social & $\begin{array}{l}\text { Regista-se uma perda de atividades ligadas ao papel social que desempenhavam no passado } \\
\text { (trabalho, vida social, vida intima e familiar) (Henriques, 2015; Hubbard et al.,2010; Lempp } \\
\text { et al., 2006). }\end{array}$ \\
\hline
\end{tabular}


Quando os elementos identitários se encontram afetados, a perceção de si pode surgir desintegrada e/ou incompleta e a saúde mental pode ser posta em causa, existindo, por isso, 'risco de perturbação da identidade pessoal', tal como é descrito NANDA - North American Nursing Diagnosis Association 2015-2017. Este risco pode converter-se num problema real, quando a referida perturbação ocorre de forma persistente, ou seja, quando permanece uma "incapacidade em manter uma perceção do self integrada e completa" (Herdman \& Kamitsuru, 2014, p. 268) .

\section{Sentimento de Si na Doença Crónica}

A experiência de viver com doença crónica afeta a identidade pessoal de uma forma disruptiva: Sentimento de perda; Sentimento de estranheza: 'o outro eu'; Sentimento de insegurança; Sentimento de luta permanente pela normalidade; Sentimento de isolamento.

\section{Sentimento de perda.}

$\mathrm{Na}$ perceção dos participantes, viver com doença crónica é uma experiência de permanente ameaça e de perda do Self e da identidade passada (Golub et al., 2013) e de perda de qualidade de vida (Adams et al., 2015; Baumgartner, 2007; Golub et al., 2013). Perde-se a motivação para as diferentes atividades (Guise et al., 2010), a independência (Henriques, 2015; Hubbard et al., 2010; Lempp et al., 2006), a autoestima (Reynolds \& Prior, 2006), a aparência e a autoimagem (Hubbard et al., 2010; Lempp et al., 2006; Reynolds \& Prior, 2006). Perdem-se relações íntimas e afeto, pela frequente falta de energia, pelas limitações e pelas mudanças na aparência. Perdem-se relações com a família, no trabalho e com os grupos de recreação, o que se traduz na perda dos diferentes papéis sociais (Lempp et al., 2006). $A$ adesão ao regime terapêutico pode igualmente conduzir a um conjunto de perdas de hábitos diários (Adams et al., 2015; Shepherd, 2010).

\section{Sentimento de estranheza: 'o outro eu'.}

Com a doença crónica a experiência do corpo alterase, pois corpo e Self deixam de ser percecionados como unidade.

O corpo, que está permanentemente impedido de agir de acordo com a identidade habitual, passa a ser percecionado como desconhecido, o que leva a flutuações no sentimento de si, com momentos de desilusão (Adams et al., 2015; Henriques, 2015; Lempp et al., 2006; Shepherd, 2010).
Existe o sentimento de que se está preso no corpo que não se reconhece, nem se deseja, um 'outro eu' (Henriques, 2015) que passa a estar sujeito à dependência, a uma vida restritiva (Henriques, 2015; Hubbard at al., 2010; Lempp et al., 2006), a um regime terapêutico imposto (Adams et al., 2015; Henriques, 2015; Shepherd, 2010) e a novas ações de autocuidado não-familiares e frequentemente ineficazes (Henriques, 2015; Hubbard at al., 2010; Lempp et al., 2006). Os sonhos, os planos, os objetivos e até a longevidade estão constantemente sob ameaça (Henriques, 2015). Assiste-se a uma grande dificuldade em manter as habituais atividades de vida diária, pelas limitações que o corpo enfrenta e pela perda de funcionalidade (Guise et al., 2010; Hubbard et al., 2010). O espaço e o tempo em que ocorrem as atividades diárias passam a ter significados diferentes (Henriques, 2015). Verifica-se a necessidade de construir um novo ritmo, com necessidade de introduzir pausas regulares, pela fadiga instalada (Henriques, 2015). A performance ocupacional e laboral ficam afetadas (Baumgartner, 2007; Lempp et al., 2006), assim como o perfil emocional e de personalidade, que adquirem atributos com os quais a pessoa não se reconhece (Lempp et al., 2006).

\section{Sentimento de insegurança.}

Verifica-se imprevisibilidade nos sintomas, o que faz com que a pessoa perceba a morte mais próxima (Henriques, 2015; Hubbard et al., 2010). As perdas são, por isso, acompanhadas de preocupações com o futuro (Henriques, 2015; Lempp et al., 2006) e por sentimentos de insegurança (Henriques, 2015; Shepherd, 2010), frustração (Guise et al., 2010), perda de espontaneidade e controlo sobre a vida (Henriques, 2015).

\section{dade.}

\section{Sentimento de luta permanente pela normali-}

$\mathrm{Na}$ convivência com a doença crónica self e identidade pessoal sofrem um processo de mudança (Baumgartner, 2007; Guise et al., 2010; Reynolds \& Prior, 2006). Neste processo as várias áreas da vida carecem de ser redefinidas, o que acontece com diferentes graus de reconciliação com a identidade passada ou de resignação passiva (Luyckx K. , et al., 2008).

É preciso integrar novas práticas no quotidiano, sejam pelas limitações que afetam as diferentes atividades de vida, sejam pelo novo regime terapêutico (Adams et al., 2015; Shepherd, 2010). Assiste-se a uma reorganização da rotina e capacidades mobilizadas no quotidiano (Adams at al., 2015). 
Neste processo de mudança, a pessoa reavalia diferentes áreas da sua vida (Baumgartner, 2007; Henriques, 2015), desenvolvendo uma luta permanente para ser normal (Adams et al., 2015; Baumgartner, 2007; Hubbard et al., 2010; Shepherd, 2010).

O contexto em que a pessoa está inserida pode ser um facilitador ou tornar-se um obstáculo neste processo (Adams et al., 2015; Guise et al., 2010;). A (re)construção da identidade está muito dependente de processos internos de descentralização da doença, em que a pessoa, na procura de equilíbrio e de novas formas de autocuidado, aprende a entender-se como sendo mais do que a própria doença (Baumgartner, 2007; Henriques, 2015; Lempp et al., 2006). Na procura de mestria e de competência no funcionamento quotidiano, a pessoa passa conseguir diferenciar a identidade antiga da identidade que está em (re)construção (Adams et al., 2015; Henriques, 2015; Reynolds \& Prior, 2006; Shepherd, 2010).

\section{Sentimento de isolamento.}

Viver com doença crónica interfere na interação com os outros e a autoperceção de desempenho eficaz do papel social (Baumgartner, 2007; Golub et al., 2013; Guise et al, 2010; Henriques, 2015; Lempp et al., 2006; Luyckx K. , et al., 2008). Esta é uma dimensão importantíssima, pois a reconstrução da identidade pessoal está muito dependente da visão dos outros. As diferentes limitações trazem agregadas o sentimento de inutilidade (Hubbard et al., 2010). Quando se tenta preservar a imagem de individuo socialmente ativo, coloca-se em risco a saúde física e mental (Baumgartner, 2007; Golub et al., 2014; Lempp et al., 2006; Luyckx K. , et al., 2008). Os doentes crónicos passam a ter receio da exposição pública (Henriques, 2015), pelo estigma e discriminação a que são sujeitos (Baumgartner, 2007; Golub at al., 2013; Guise et al., 2010; Lempp et al., 2006), o que conduz a situações de redução da participação social (Lempp et al., 2006).

\section{Fases da (Re)construção da Identidade Pessoal}

Para que ocorra adaptação à doença crónica, é necessário que a pessoa desenvolva mudanças de comportamento e aprendizagem de novos hábitos. Recorrendo ao Modelo Transteórico de Mudança de Comportamento (Prochaska et al., 2008), identificou-se os atributos que permitem caracterizar cada fase da mudança comportamental até à (re)construção da identidade pessoal.

\section{Identidade antiga.}

Nesta fase (de pré-contemplação) a pessoa não tem consciência da sua doença crónica ou não conhece as suas implicações, apesar de, por vezes, conseguir antecipar o seu diagnóstico (Baumgartner, 2007).

Nesta etapa, a doença crónica não tem a importância suficiente para que seja iniciada uma tentativa de mudança de comportamento, pelo que a pessoa enceta diferentes tentativas para retomar as atividades que integram a identidade pessoal anterior (Henriques, 2015; Lempp et all., 2006). As pessoas lutam para fazer parte da sociedade, colocando até em risco a sua saúde (Henriques, 2015).

\section{Ameaça à identidade.}

Esta fase (de contemplação) inicia-se com o momento em que é atribuído um diagnóstico médico (Adams et al., 2015; Baumgartner, 2007; Hubbard et al., 2010; Lempp et al., 2006; Shepherd, 2010).

Neste estágio a pessoa clarifica as suas suspeitas, reconhece o problema e inicia uma discussão (interna e/ou externa) acerca da necessidade de mudar comportamentos.

Apesar de conhecer a direção que pretende tomar, de identificar os prós e os contras em manter comportamentos ligados à identidade atual, há uma certa hesitação em enfrentar a mudança. Um menor sentimento de identidade implicará mais tempo nesta fase e um maior sentimento de identidade conduzirá, pelo contrário, a uma mais rápida transição para as fases seguintes (Prochaska et al., 2008).

\section{Negociação identitária.}

Esta fase (de preparação) é um momento de desconstrução cognitiva, afetiva e psicomotora da identidade atual, para introduzir novos elementos identitários (Shepherd, 2010).

Nesta etapa existe uma clara consciência da necessidade de mudança e uma determinação para iniciar esse processo num futuro próximo. A doença passa a ser integrada na definição de si, o que permite aos indivíduos construírem um conjunto de estratégias de coping adaptativas para lidar com a cronicidade (Henriques, 2015; Luyckx K. , Seiffge-Krenke, Schwartz, Goossens, Hendrieckx, \& Groven, 2008). A doença traz tempo de reflexão, o que leva a uma reavaliação dos hábitos e limites (Henriques, 2015), a uma reinterpretação do sentido da vida e a uma redefinição de objetivos (Hubbard et al., 2010) e desejos (Adams et al., 2015 
O desejo de ser independente e autónomo, não se constituindo uma sobrecarga para os outros, é o que mais acompanha estas pessoas (Henriques, 2015; Hubbard et al., 2010; Lempp et al., 2006). Passa a existir um planeamento meticuloso para evitar a exposição pública (Henriques, 2015).

\section{(Des/re)construção da identidade.}

Nesta fase (de ação) dão-se início a um conjunto de comportamentos observáveis que evidenciam uma mudança comportamental e uma reorganização do quotidiano. A pessoa vai construindo os seus novos hábitos, adaptando os que faziam parte da identidade antiga ou eliminando outros (Hubbard et al., 2010; Henriques, 2015). Esta (des/re)construção da identidade assenta num trabalho biográfico de ajuste da forma de se autocuidar, tendo em conta o que faz sentido a cada um (Henriques, 2015). A ausência de sentido de um novo hábito pode motivar uma recaída no comportamento antigo (Henriques, 2015), o que significa um retrocesso para a fase de contemplação. Estas situações reduzem o sentimento de identidade, dificultam o sentimento positivo do self e estão associadas a sintomas depressivos e a um pior controlo sintomático (Golub et al., 2014; Guise et al., 2010; Luyckx K. , et al., 2008). É nesta fase que alguns doentes passam a integrar grupos de ajuda ou associações de doentes (Baumgartner, 2007; Henriques, 2015) e que se verificam comportamentos de adesão ao regime terapêutico proposto (Adams et al., 2015; Baumgartner, 2007; Shepherd, 2010).

\section{Identidade reconstruída.}

Na última fase, (de manutenção), a pessoa mantém comportamentos procurando não perder o que foi conquistado na fase anterior. Mantém-se o desejo de mudança e o esforço de prevenir recaídas que levem ao comportamento indesejado. Nesta etapa as pessoas compreendem melhor as suas prioridades, desenvolvem novos talentos ou preservam os elementos identitários que têm maior significado para si (Henriques, 2015). Demonstram grande capacidade de adaptação interna e externa às novas limitações que surgem. É uma fase que se caracteriza pelo aumento da autoestima, pela integridade pessoal e por um incremento no conhecimento (Prochaska et al., 2008). Há a perceção de crescimento pessoal e um sentimento de reconstrução pessoal (Golub et al., 2013; Golub et al., 2014). Existe um sentimento de continuidade, de conexão com o self prévio à doença (Henriques, 2015) e de normalidade (Shepherd, 2010).

\section{CONCLUSÕES E IMPLICAÇÕES PARA A PRÁTICA CLIINICA}

Esta é a primeira revisão da literatura conhecida sobre a identidade pessoal na experiência de conviver com a doença crónica. A análise da amostra bibliográfica permitiu identificar os elementos identitários postos em causa na doença crónica, o sentimento de si na doença crónica, bem como as fases de reconstrução da identidade pessoal na doença crónica. Cada uma destas categorias foi descrita através de um conjunto de subcategorias. As pessoas procuram naturalmente definir e expressar a sua identidade pessoal. A procura de unidade, coerência consigo própria e consistência, pode estar perturbada pela convivência com a doença crónica. Vários elementos identitários, como o grau de atividade, a capacidade física ou o grau de dependência, ficam perturbados, podendo conduzir a sentimentos de perda, estranheza, insegurança ou isolamento. A pessoa percorre diferentes estádios até reconstruir a sua identidade pessoal: identidade antiga, ameaça à identidade, negociação identitária, (des/re)construção da identidade e identidade reconstruída.

Esta revisão integrativa permite ampliar a compreensão do processo de reconstrução da identidade pessoal na doença crónica, dando subsídios importantes para uma enfermagem assente numa apreciação holística e desafiando para uma prática de cuidados onde a saúde mental ganha transversalidade. Esta orientação para o que constitui a identidade pessoal do cliente de cuidados de enfermagem desafia os enfermeiros a assumirem a responsabilidade de expressar autenticidade e ser parte do mundo-vivido do cliente, no sentido de perceber que elementos identitários são importantes preservar no processo de reconstrução identitária.

Os resultados desta revisão deixam também contributos para estruturar investigações futuras sobre a reconstrução da identidade pessoal na doença crónica, principalmente ao nível da clarificação das diferentes fases deste processo, bem como na definição de intervenções de enfermagem ajustadas às diferentes perdas. Abrem igualmente a possibilidade de desenvolver instrumentos de medida que permitam quantificar a perturbação (ou risco de) da identidade pessoal na doença crónica, bem como a eficácia das intervenções de enfermagem. Esta revisão integrativa da literatura apresenta algumas limitações metodológicas, que devem ser consideradas. A categorização criada para apresentar os resultados muda em função da interpretação do investigador, o que inviabiliza a replicação do estudo. 
Também o facto de a revisão ter sido realizada apenas por um investigador pode ter tornado a análise mais pobre. Os resultados são, ainda, indicativos, mas não generalizáveis.

\section{REFERÊNCIAS BIBLIOGRÁFICAS}

Adams, W. E., Todorova, I. G., Guzzardo, M. T., \& Falcón, L. M. (2015). The problem here is that they want to solve everything with pills': medication use and identity among Mainland Puerto Ricans. Sociology of Health \& Illness, 37(6), 904-919.

Baumgartner, L. M. (2007). The incorporation of the HIV/AIDS identity into the self over time. Qualitative Health Research, 17(7), 919-931.

European Union. (2016). European framework for action on mental health and wellbeing. Joint Action on Mental health and wellbeing. Brussel: União Europeia.

Golub, S. A., Gamarel, K. E., \& Rendina, H. J. (2014). Loss and growth: Identity processes with distinct and complementary impacts on well-being among those living with chronic illness. Psychology, Health \& Medicine, 19(5), 572-579.

Golub, S. A., Rendina, H. J., \& Gamarel, K. E. (2013). Identity-related growth and loss in a sample of HIVpositive gay and bisexual men: initial scale development and psychometric evaluation. AIDS And Behavior, $17(2), 748-759$.

Guise, J., McKinlay, A., \& Widdicombe, S. (2010). The impact of early stroke on identity: A discourse analytic study. Health, 14(1), 75-90.

Henriques, H. (2015). Experiência do autocuidado e identidade pessoal: Um estudo fenomenológico com pessoas idosas que vivem com doença pulmonar obstrutiva crónica (Tese Doutoramento). Universidade católica Portuguesa, Instituto de Ciências da Saúde, Lisboa.

Herdman, T. H., \& Kamitsuru, S. (2014). NANDA International Nursing Diagnosis: Defenitions \& Classification, 2015-2017. Oxford: Wiley Blackwell.

Hubbard, G., Kidd, L., \& Kearney, N. (2010). Disrupted lives and threats to identity: the experiences of people with colorectal cancer within the first year following diagnosis. Health, 14(2), 131-146.
Karnilowicz, W. (2011). Identity and psychological ownership in chronic illness and disease state. European Journal Of Cancer Care, 20(2), 276-282.

Lempp, H., Scott, D., \& Kingsley, G. (2006). The personal impact of rheumatoid arthritis on patients' identity: a qualitative study. Chronic Illness, 2(2), 109-120.

Luyckx, K., Seiffge-Krenke, I., Schwartz, S. J., Goossens, L. W., Hendrieckx, C., \& Groven, C. (2008). Identity development, coping, and adjustment in emerging adults with a chronic illness: The sample case of type 1 diabetes. Journal of Adolescent Health, 43(5), 451-458.

Mathews, E. (2013). Personal identity and mental health. In D. C. Thomasma, N. Weisstub, \& Hervé (Eds.), Personhood and health care (pp. 203-212). Paris: Springer Science \& Business Media.

Meleis, A. I., Sawyer, L. M., Im, E., Messias, D. K., \& Schumacher, K. (2000). Experiencing Transitions: An Emerging Middle-Range Theory. Advances in Nursing Science, 23, 12-28.

Nordenfelt, L. (2009). Dignity in care for older people. West Sussex, England: Wiley-Blackwell.

Prochaska, J. O; Redding, C. A., \& Evers, K. E. (2008) The transtheoretical model and stages of change. In $\mathrm{K}$. Glanz, F. Lewis \& B. Rimer (Eds.), Health Behavior and Health Education: Theory, Research, and Practice (4th ed.) (pp 97-122). San Francisco: Jossey Bass Publishers.

Rafael, H. (2012). Identidade Pessoal. Que significações do conceito no âmbito dos cuidados de enfermagem?. Livro de resumos da $12^{\text {a }}$ Conferência Internacional de Investigação em Enfermagem. Enfermagem baseada na evidência: estratégias de investigação (pp. 23). Associação Portuguesa de Enfermeiros: Lisboa.

Reynolds, F., \& Prior, S. (2006). The role of art-making in identity maintenance: case studies of people living with cancer. European Journal of Cancer Care, 15(4), 333-341.

Shepherd, M. (2010). Stopping insulin injections following genetic testing in diabetes: Impact on identity. Diabetic Medicine: A Journal of The British Diabetic Association, 27(7), 838-843.

Souza, M., Silva, M., \& Carvalho, R. (2010). Revisão integrativa: O que é e como fazer. Einstein, 8 (1), 102-106. 